\title{
SIMULATION ANALYSIS FOR REDUCING QUEUES IN MIXED-PATIENTS' OUTPATIENT DEPARTMENT
}

\author{
Wijewickrama, A. K. A. \\ Nagoya University, Graduate School of Economics and Business Administration \\ Furo-cho, Chikusa-ku, Nagoya, Aichi 464-8601, Japan

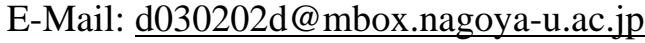

\begin{abstract}
Customers desire short waiting times whereas service providers want to maximize resources utilization. Long waiting time is not uncommon in many service organizations and it is familiar especially in outpatient departments. To make the study more realistic, some assumptions were removed and uses an animated simulation model for a mixed-patient type environment in an outpatient department. A special purpose data generator is designed to explore bottlenecks in consultation rooms. Four appointment scheduling rules and their possible combinations are evaluated in two steps. First, experimentation concentrates on appointed and non-appointed patients. Second, it considers new patients in addition to those two categories. It is revealed that the rule which records the lowest waiting time is not feasible due to the high portion of server idle time. The rule that shows the lowest server idle time is not viable due to increased waiting time. It is possible to find the best rule which lies between these two times in a mixed-patient type environment.

(Received in May 2005, accepted in February 2006. This paper was with the author 3 months for 2 revisions.)
\end{abstract}

Key Words: Appointment Scheduling, Outpatient Department, Discrete Event Simulation

\section{INTRODUCTION}

Soaring health care costs and greater emphasis on preventative medicine have compelled researchers to examine new ways to reduce costs and improve the efficiency in outpatient services. In recent years, with a considerable increase in the movement of health care from in-patient cases to outpatient cases, outpatient services have gradually become an essential component in health care. In many outpatient departments of Japan, the long waiting times for treatment followed by short consultations have long been a major complaint of patients. Such complaints are often phrased as "waiting for three hours to be seen for three minutes". Over the years, this disproportionately long waiting time in the consultation room has been the focus of research among academicians and practitioners. Most researchers have stressed that the major reason for long waiting times is the poor patient appointment system in place.

Traditionally, the medical profession highlighted that a physician's time is more valuable than a patient's so that the appointment schedule (AS) was designed to minimize physicians' idle time overlooking patients' waiting time. This is no longer valid in today's consumer oriented society. Today, customers use waiting time as a decisive factor in choosing a service provider. Therefore, idle time of both parties must be considered in designing an AS although these two objectives are contradictory to each other. This study aims to develop such an appointment system in a real world healthcare organization: a mixed patients' outpatient department in Japan. 


\section{BRIEF LITERATURE REVIEW}

The types of appointment systems range from single-block appointments on the one extreme to individual appointments on the other. Most of the appointment systems have concentrated on modifying and combining these two systems into different forms. Any combination in the appointment interval, block size and initial block create an AS rule.

The single-block system assigns all patients to arrive in a block at the beginning of the clinic session. It allocates a "date" rather than a specific appointment time [1]. Such a system was in the past used by most hospitals. The single-block system creates long waiting time for patients but shortens idle time for doctors. This unfairness led to critical research in AS rules, so that an individual-block system emerged.

The individual-block/fixed-interval system gives unique appointment times for patients staggered evenly over the clinic session [2, 3].

The third rule, individual-block/fixed-interval with an initial block system is similar to the second rule, but the number of patients assigned to initial block is greater than one. Bailey [4] introduced this rule to the AS literature.

Following an analytical approach, Soriano [5] advocated the multiple-block/fixed-interval rule to the AS literature recommending patients to schedule two at a time with an interval of twice the consultation time.

In another study [6] investigated the multiple-block/fixed-interval with an initial block rule, introducing an initial block to the above rule. The simulation model developed for the ear, nose, and throat (ENT) clinic was validated by comparing results with actual data.

The Variable-block/fixed-interval assigns a different number of patients during the clinic session [7, 8].

The Individual-block/variable-interval rule calls patients individually with unequal appointment intervals. Introducing this rule to the literature, Ho and Lau [9] concluded that a variable-interval appointment-scheduling system designed to reduce patient waiting time performs well in most environmental conditions. Yang et al., [10] analyzed this rule simulating [9]'s related variable-interval rules. Vanden Bosch and Dietz [11] also addressed this rule in their study.

\section{SYSTEM DESCRIPTION}

\subsection{Description of the Outpatient Department}

Overall layout of the outpatient department is shown in the Fig. 1. The department under study operates from 8.30 to 17.30 during weekdays with four different types of patient visits: appointed patients, non-appointed patients, new patients and patients who come for a medical test. Similar to prevailing hospital outpatient departments in Japan, the largest percentage, a 65 percent of the total patient mix, consists of patients with appointments. Non-appointed and new patient visits are 14 and 11 percent respectively, and the rest belongs to the patients who come for a medical test (i.e. 10 percent). Fig. 2 shows the generalized patient flow diagram.

All patients have to go to the reception desk for submission of their hospital card and required papers, and if necessary, for initial screening. New patients have to go to the new patient desk for filling-out applications, showing their health insurance certificate and obtaining the hospital card. Patients who come for a medical test can go directly to the relevant test room after meeting the receptionist. Depending on the sickness or the availability of the pre-appointed test, the required patients have to do lab tests such as urine and blood tests or go to the X-ray room. Patients who make an appointment have priority over those 
who non-appointed patients for a consultation. A separate physician has been allocated to care for new patients.

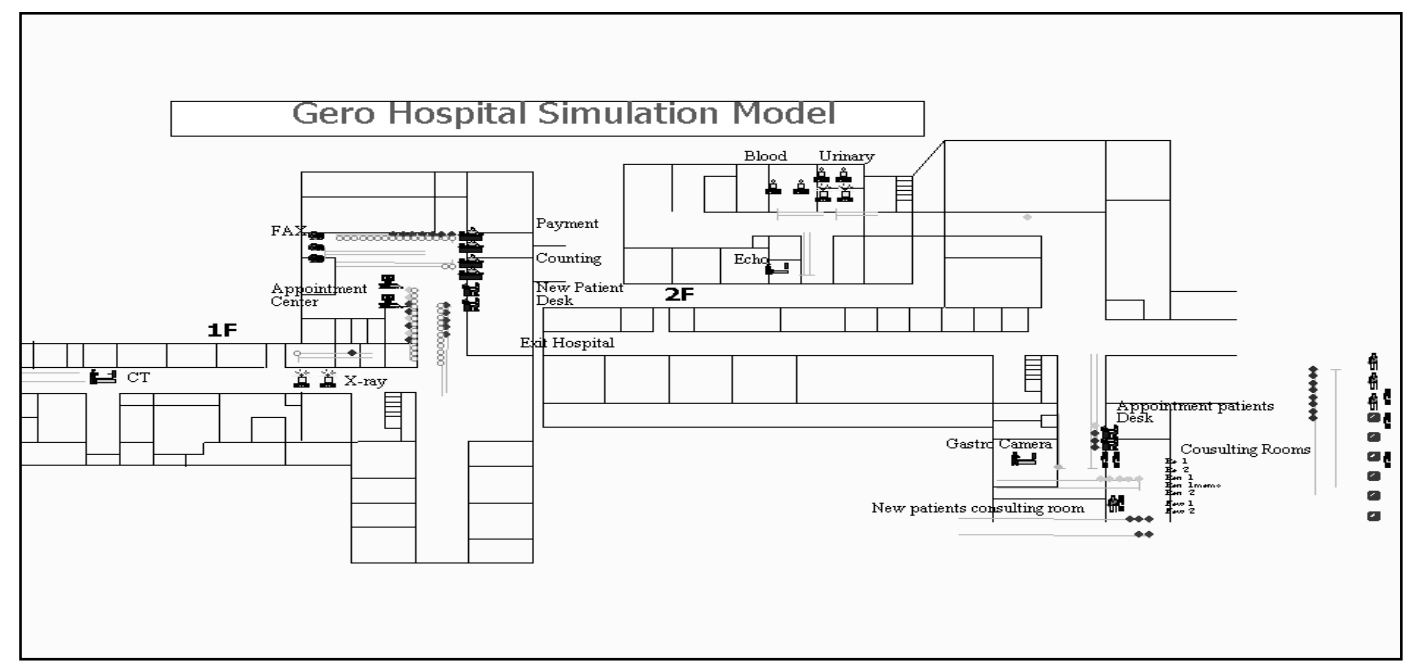

Figure 1: Layout of the Outpatient Department.

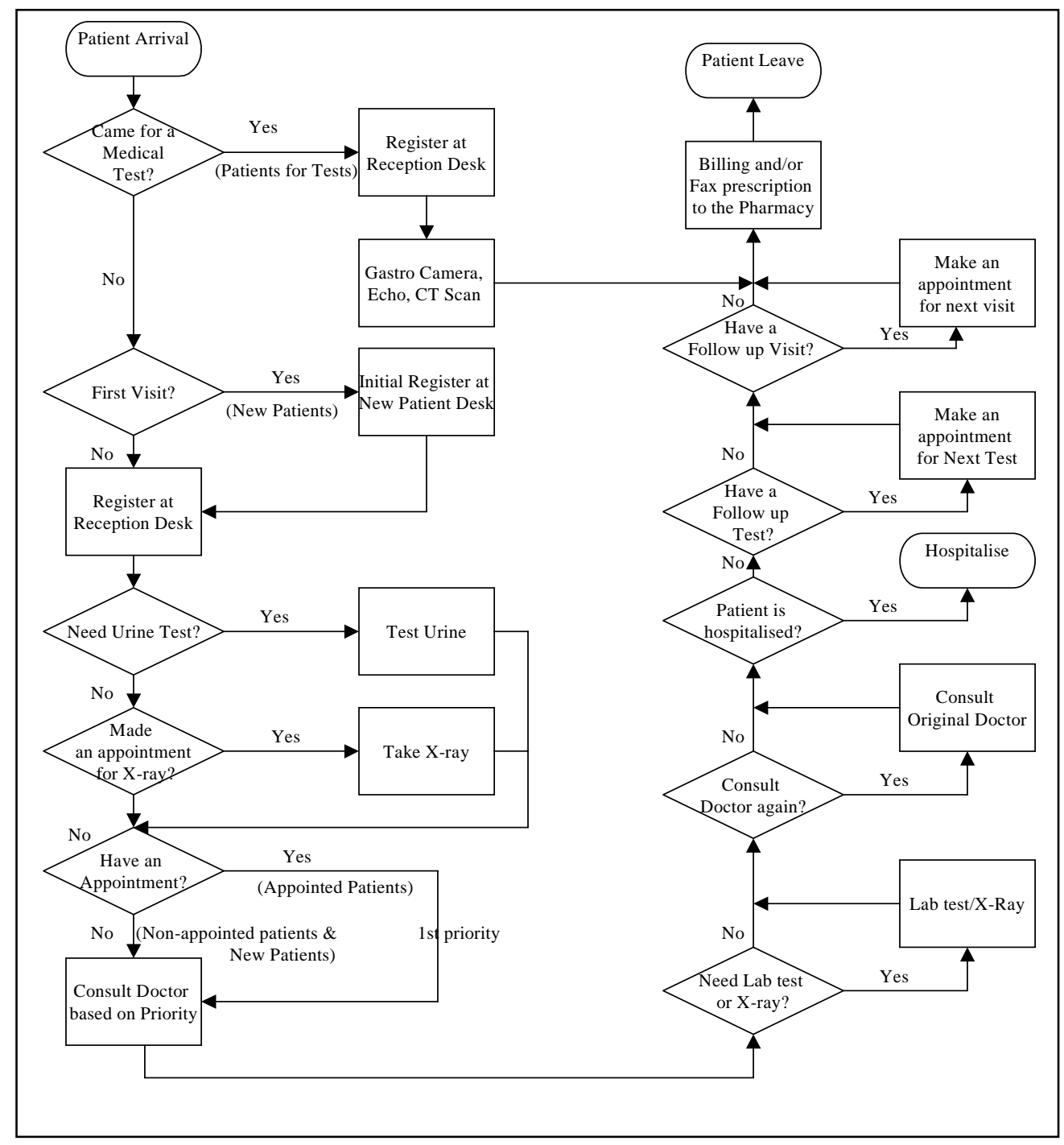

Figure 2: Patient Flow. 
During the consultation with the physician he decides whether the patient needs to be sent for a laboratory or X-ray. Patients who undergo a laboratory test or X-ray, have to consult the same physician before leaving the hospital. After the second assessment, patients can leave the hospital after making appointments for follow-up tests and/or visits. Patients can order needed drugs from a pharmacy by faxing the prescription after settling the bill at cashier. Based on the severity of the sickness, the physician may decide to discharge or hospitalise the patient.

\subsection{Performance measures}

This study employs a discrete event simulation methodology in an outpatients department in Gero prefectural hospital in Gifu, Japan. The objective of this study is to minimize patients' waiting time by keeping doctor idle time as low as possible. The consultation rooms cater their services for three types of patients. These three categories of patients were combined when measuring performance. Accordingly, two primary performance measures were considered in this study:

(i) Weighted average of all three types of patients' waiting time (WAPWT - including service time) and

(ii) Weighted average of all three types of patients' server idle time (WASIT).

Liu and Liu [8] and Wang [12] considered patient waiting time (including service time) as the main performance measure in their studies. However, some other researchers have used both patient waiting time (including or excluding service time) and physician idle time to measure performance (see [2, 3, 8, 9, 10]).

It is clear that most of the studies have used discrete event simulation methodology instead of using analytical methods due to the inherent complexities of the system. However, this subject been researched for more than fifty years, and a big gap still exists between theory and practice. The major reason for this phenomenon is the unrealistic assumptions made on the model development process. Previous studies that have made use of ASs have ignored at least one of the following:

- Walk-ins and other patients such as patients, who come for medical tests,

- Inter-related subunits that use outpatients such as laboratories, receptionist, payment counters,

- Other patients who use facilities which also used by outpatients,

- Second time consultation,

- Doctors' idle time and realistic doctors schedules that not disrupt day-to-day activities of the hospital.

The present study emphasizes these issues to achieve the above-mentioned objective.

When inputs vary (e.g., patient arrival) and service times are uncertain (e.g., consultation time), it cannot use a deterministic single value. In a health care facility, most entities and resources are in the form of a physical being and are not like machine-parts in a manufacturing setting. Modelling the human interactions of a patient, physician, and nurse is enormously complex due to the inherent nature of the variability. Patients and staff may not be punctual. The doctor may not be able to identify the medical problem precisely and diagnostic time is unpredictable. Patients may go home due to the long queue time without consulting the doctor. Patients may tend to talk unnecessarily for a long time and argue with staff members. The doctor may have to attend emergency situations keeping appointed patients waiting. McGuire [13] pointed out the reason for variability inherent in the system as the involvement of "intelligent entities". 


\subsection{Data collection}

Data was collected via interviewing administrators, physicians, nurses and other clerical personnel, computer-recorded files, and by observation. Staff and appointment schedules, inter-arrival times of patients, steps involved in each sequence or route, the routine probabilities, process time, sub units and the relationships among sub units in each sequence for each patient category, and time taken to walk between two subunits represent the data collected from these sources. Accordingly, 31 distinct sequences were identified. Average process and delay time for each unit is summarized in the Table I.

Table I: Process and Delay Time.

\begin{tabular}{|c|c|}
\hline Items & Time (min.) \\
\hline New patient receptionist & $\operatorname{EXPO}(6)$ \\
Patient receptionist & $\operatorname{EXPO}(1.333)$ \\
\hline Consultation & \\
Appointment \& Non-appointed-1st time & $\operatorname{TRIA}(2,3.67,8)$ \\
Appointment \& Non-appointed-2nd time & $\operatorname{EXPO}(2)$ \\
New-1st time & $\operatorname{TRIA}(7,15,20)$ \\
New-2nd time & $\operatorname{UNIF}(3,5)$ \\
\hline Blood test & $\operatorname{EXPO}(3)$ \\
Waiting for lab test report & $\operatorname{TRIA}(7,15,25)$ \\
X-ray & $\operatorname{EXPO}(4)$ \\
Payment & $\operatorname{EXPO}(0.58333)$ \\
Prescription fax & $\operatorname{EXPO}(0.36667)$ \\
Urine test & $\operatorname{EXPO}(3)$ \\
Follow up test appointment & $\operatorname{UNIF}(2,3.5)$ \\
Gastro Camera & $\operatorname{TRIA}(10,20,30)$ \\
Abdomen Echo & $\operatorname{UNIF}(7,15)$ \\
CT test & $\operatorname{EXPO}(0.75)$ \\
Follow up appointment & $\operatorname{EXPO}(0.3)$ \\
Billing &
\end{tabular}

The total number of visits was over one thousand patients per week and the inter-arrival time of each patient category, except appointed patients, was estimated according to exponential distribution. Patients who came outside of the clinic hours were simply removed from the model. All the patients who arrived during the opening hours were able to obtain services within the present arrival level. The existing AS was prepared base on the availability of physicians and their capacities. The number of patients coming in each half an hour deviates from 17 to 5, within the clinic hours from 9.00 to 14.30.

Among nine physicians scheduled for a week, three physicians engage in consultation with these two categories of patients in a given day. One physician has been allocated to consult with new patients. Six nurses are on duty and they work in the medical test appointment centre, examination rooms, and consultation rooms. The working time of each physician and nurse is based on schedules, including breaks. 


\section{SIMULATION MODEL}

\subsection{Modelling features}

A simulation model for the outpatient department was created using the simulation package Arena [14]. For the purpose of validating the model and conducting experiments in bottleneck situations in the consultation rooms of the existing system, a special-purpose data-generator was designed using Visual Basic for Application (VBA) in Arena. This automates the Arena model according to user request while generating experimental data. The initial VBA user form represents the existing state and the user is allowed to change the total number of patients and the patient mix. This kind of data generator has been used in a previous study examining patient flows in an emergency department of a general hospital [15]. At the beginning of the simulation run, the user is allowed to change the basic input parameters of the model being used as shown in following Fig. 3.

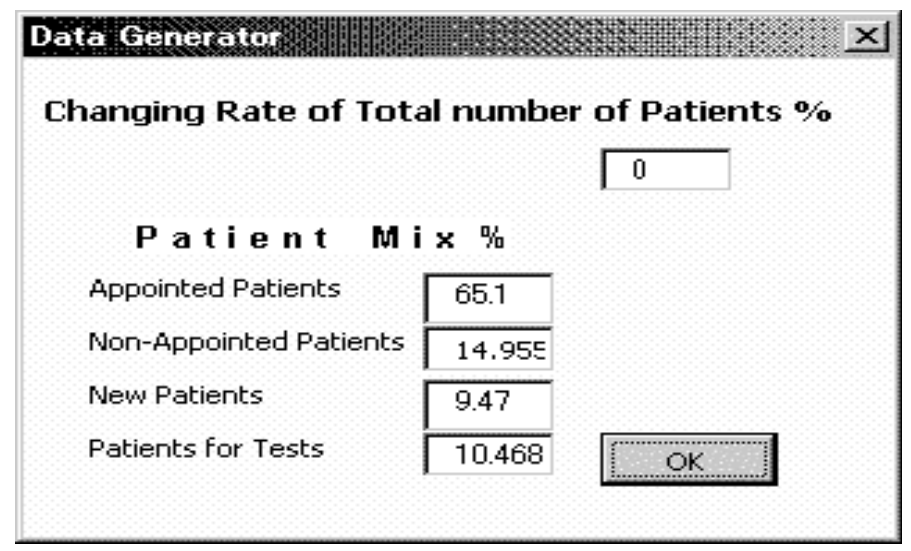

Figure 3: Data Generator user form.

According to a user's request, the program rearranges the AS, sets new inter-arrival time of other patients, and initializes variables such as physicians' capacities over the replications. In short, under any specified condition, the VBA code alters the modules of the model without touching the model.

\subsection{Verification and validation of the model}

The model validation is the lengthiest step of the actual simulation model and McGuire highlights that this step in the project usually causes the most headaches in health care simulation [13]. A number of techniques were used to verify and validate the model.

First, animation screen together with dynamic statistics and graphs provided a general view of the system behaviour. The researcher closely examined to verify whether the animation imitates the actual system. Second, in the case of face validity, a team consisting 15 members evaluated the system and their valuable comments helped to augment the model. Third, varying behaviour of some performance measures were examined by adjusting the patient arrivals with incremental percentages. Ten percent incremental arrivals from 0 to 50 percent were observed and the impact on number of waiting patients and the number of patients who cannot obtain the service before 17.30 (i.e. over time (OT) need patients) was examined, as shown in Fig. 4. 


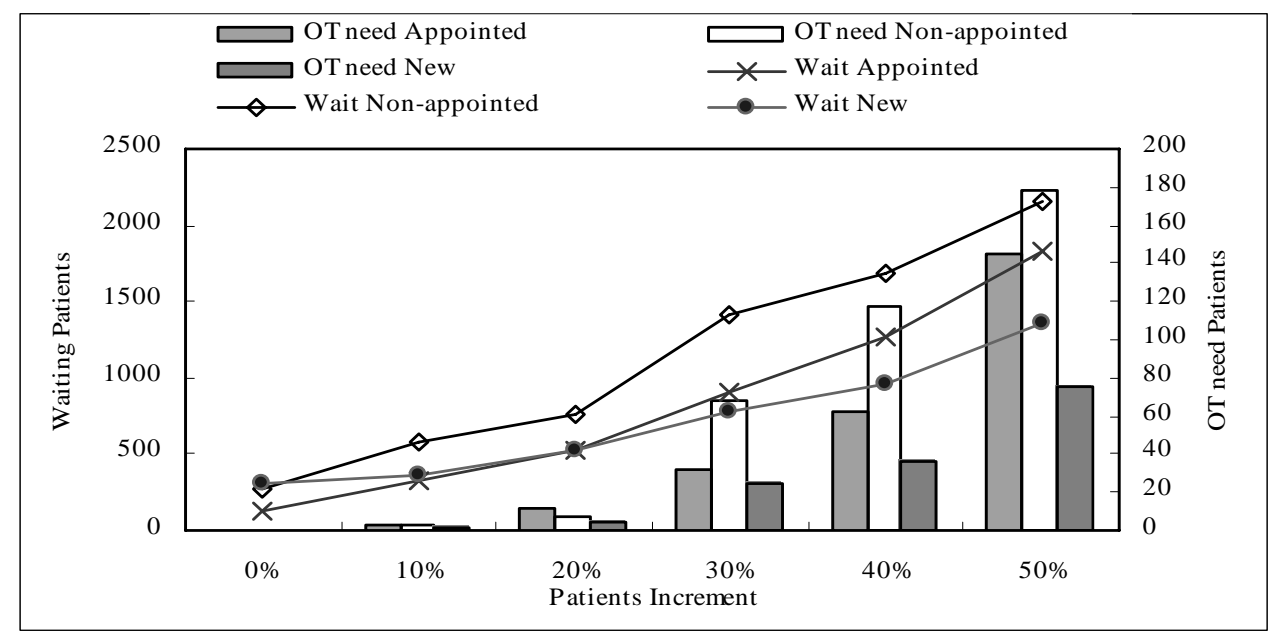

Figure 4: Impact of incremental arrival.

Fig. 4 shows that the number of waiting patients and the number of OT need patients are increasing as incremental arrival complying with the consistency of the model.

In addition to the above techniques, execution of tracing, self-documentation and checking the computerized representation by another developer were also used to build a more realistic model. (See [16] for more details on verification and validation techniques used in a different application of a hospital simulation model).

Based upon the results from verification and validation techniques applied in testing, the model provided realistic predictions for the system behaviour under the various experimentations illustrated in the next part of this paper.

\subsection{Simulation analysis}

Results from the simulation model reveal that patients have to wait a long time to consult a physician even though it may take only a few minutes to examine a patient. According to the Table II, the proportion of waiting time varies from 65-98 percent. Consequently, as in Fig. 5, the total number of waiting patients in front of consultation rooms gradually increased to twenty-six patients around 12 noon, and then slowly decreased.

Table II: Consultation Queue Time and Consultation Time (minutes).

\begin{tabular}{|c|c|c|c|c|c|c|c|}
\hline $\begin{array}{c}\text { Consultation } \\
\text { Room }\end{array}$ & \multicolumn{2}{|c|}{ Appointed } & \multicolumn{3}{c|}{ Non-appointed } & \multicolumn{2}{c|}{ New } \\
\hline \multirow{3}{*}{\begin{tabular}{c} 
Consultation \\
\cline { 2 - 8 }
\end{tabular}} & $\begin{array}{c}\text { 1st } \\
\text { Queue Time }\end{array}$ & 2nd & 1st & Memo & 2nd & 1 st & 2nd \\
\hline Consultation & 4.61 & 47.91 & 51.14 & 42.26 & 66.28 & 75.73 \\
Time & $(96 \%)$ & $(91 \%)$ & $(96 \%)$ & $(98 \%)$ & $(83 \%)$ & $(94 \%)$ \\
\hline
\end{tabular}

The experimental process concentrated on the issues of excessive waiting time at the consultation room, aiming to shorten the patients' waiting time, and not complementing the idle time of the physicians.

The demand of the outpatient department can be divided into two parts: controllable and uncontrollable. The controllable part is the appointed patients as they come for treatment at a pre-determined time. Non-appointed regular patients and new patients are the uncontrollable part as they come for treatment without notice. As a result of that, the experimentation should 
be made with controllable demand. Therefore, this study contemplates on the appointed patient category, applying different types of scheduling alternatives to shorten the waiting time of all three categories of patients.

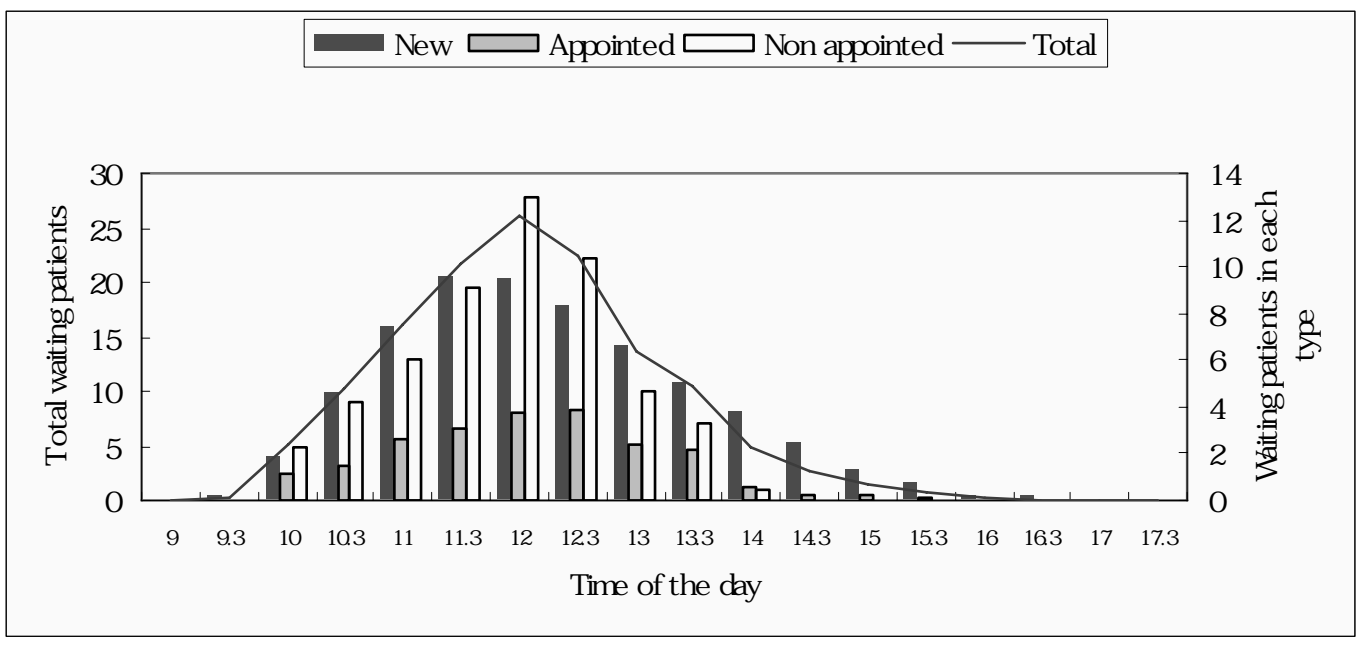

Figure 5: Total waiting patients and its composition of each patient type.

First, appointed and non-appointed regular patients are considered introducing four scheduling rules under this study (Table III).

Table III: Scheduling rules.

\begin{tabular}{|l|l|}
\hline \multicolumn{1}{|c|}{ Rules } & \multicolumn{1}{|c|}{ Description } \\
\hline $\begin{array}{l}\text { B2 } \\
\text { (Baily) }\end{array}$ & $\begin{array}{l}\text { Place two patients in the first slot, and one in each subsequent slot } \\
\text { based on average consultation time. }\end{array}$ \\
\hline Rising & $\begin{array}{l}\text { Smoothing the demand of scheduling appointment periods to } \\
\text { complement the arrival pattern of walk-in patients. }\end{array}$ \\
\hline 15MIN & Change the appointment time slot from 30 to 15 minutes. \\
\hline SPTBEG & $\begin{array}{l}\text { First priority gives for shorter processing patients in consultation a } \\
\text { physician. }\end{array}$ \\
\hline
\end{tabular}

The 15MIN rule is based on changing the time length of the appointment slot. Accordingly, the time length is shortened from 30 to 15 minutes as the number of appointment slots for a session increases. The existing scheduling system depends on the first-come-first serve basis and so does not distinguish patients on service time. Moreover, this system neither smoothens the demand of appointed patients as in Rising's rule nor shortens the appointment slot as in $15 \mathrm{MIN}$ rule. Therefore, the as-is system can be used as a benchmark to compare four scheduling rules.

Initially, these four alternative models were compared with the base model with taking the performance measure - waiting time (including service time). As there are different mean values for different alternatives, for the purpose of identifying statistically significant differences, it is suitable to use a paired-t test. Since there is no assumption of statistical independence due to employment of common random numbers for simulating two systems, the paired-t approach can be successfully used [17].

The null hypothesis $\left(H_{0}\right)$ of this analysis is that there is no difference in average waiting time between the as-is model and each alternative model. The alternative hypothesis $\left(H_{1}\right)$ is that there is a difference in average waiting time between the as-is model and each alternative 
model. The number of replications, setting the confidence level at 0.05 , is ten runs. The results are shown in Table IV.

Table IV: Waiting time of scheduling rules (minutes).

\begin{tabular}{|l|c|c|c|}
\hline Case & Appointed & Non-appointed & New \\
\hline Base & 39.13 & 76.55 & 167.05 \\
\hline B2 & $32.06^{*}$ & $97.20^{*}$ & 163.89 \\
\hline Rising & $35.69^{*}$ & $39.73^{*}$ & 171.73 \\
\hline 15MIN & $35.69^{*}$ & 72.94 & 174.21 \\
\hline SPTBEG & $33.58^{*}$ & $37.03^{*}$ & 172.36 \\
\hline
\end{tabular}

Although all rules make a significant reduction to the waiting time of appointed patients, in the case of non-appointed patients, Rising and SPTBEG highlight a substantial reduction to the waiting time. These rules do not directly affect new patients' waiting time as they consult a physician who is not involved in consulting other patients. But there is a slight variance of each case due to the indirect cause as the nature of interactions among sub-systems.

Secondly, it was considered a possible combination of the above rules. The paired-t test results are shown in Table V. It is seen that all combined pairs make a significant reduction to the waiting time of appointed and non-appointed regular patients. Especially, the cases which use SPTBEG, reduce the waiting time drastically.

An efficient frontier is derived by integrating WAPWT (appointed and non-appointed regular patients) and WASIT in each case as depicted in Fig. 6. It moves from upper left to the lower right. Points on upper left raise WAPWT and those on the lower right raise WASIT. Accordingly, B2, 15MINSPTBEG, RisingSPTBEG, and SPTBEG are inferior, as they lie on right-hand side of the frontier resulting high waiting time and/or high idle time.

Table V: Waiting time in possible combinations (minutes).

\begin{tabular}{|l|l|l|l|}
\hline Case & Appointed & Non-appointed & New \\
\hline Base & 39.13 & 76.55 & 167.05 \\
\hline 15MINRising & $33.50^{*}$ & $43.19^{*}$ & 178.25 \\
\hline B2SPTBEG & $26.31^{*}$ & $37.60^{*}$ & 165.88 \\
\hline RisingSPTBEG & $31.27^{*}$ & $30.43^{*}$ & 175.90 \\
\hline 15MINSPTBEG & $30.82^{*}$ & $35.97^{*}$ & 177.41 \\
\hline
\end{tabular}

SPTBEG, which recorded a remarkable reduction of WAPWT, shows up far from the origin due to increased WASIT. Because of the increased idle component of the SPTBEG, any combinations which were made using SPTBEG also records a high WASIT. Again, the results of the existing system display the highest WAPWT, which is a common feature in many outpatient departments. To conclude, Rising is the best principle as a single scheduling rule and 15MINRising is the best as a hybrid combination.

Considering appointed, non-appointed and new patients, the last part of the experimentation is devoted to shortening new patients' waiting time which has not yet been considered. New patients need more time for consultation than regular patients. For consultation of new patients, a separate physician has been assigned. A number of alternatives were considered from the physician's point of view as new patients belong to uncontrollable demand category. Introducing a physician for new patients adding only 1 hour capacity per day to the total number of man-hours of the base case was considered. Instead of being in the hospital throughout the day as in the base case, these two physicians consult patients in the 
morning session. In case they have free time, they can consult non-appointed regular patients too. The experimentation was undertaken to reduce the waiting time of appointed, non-appointed and new patients incorporating this alteration (denoted as P2) by the above four rules and possible combinations discussed under table IV and V. The paired-t test results are shown in Table VI.

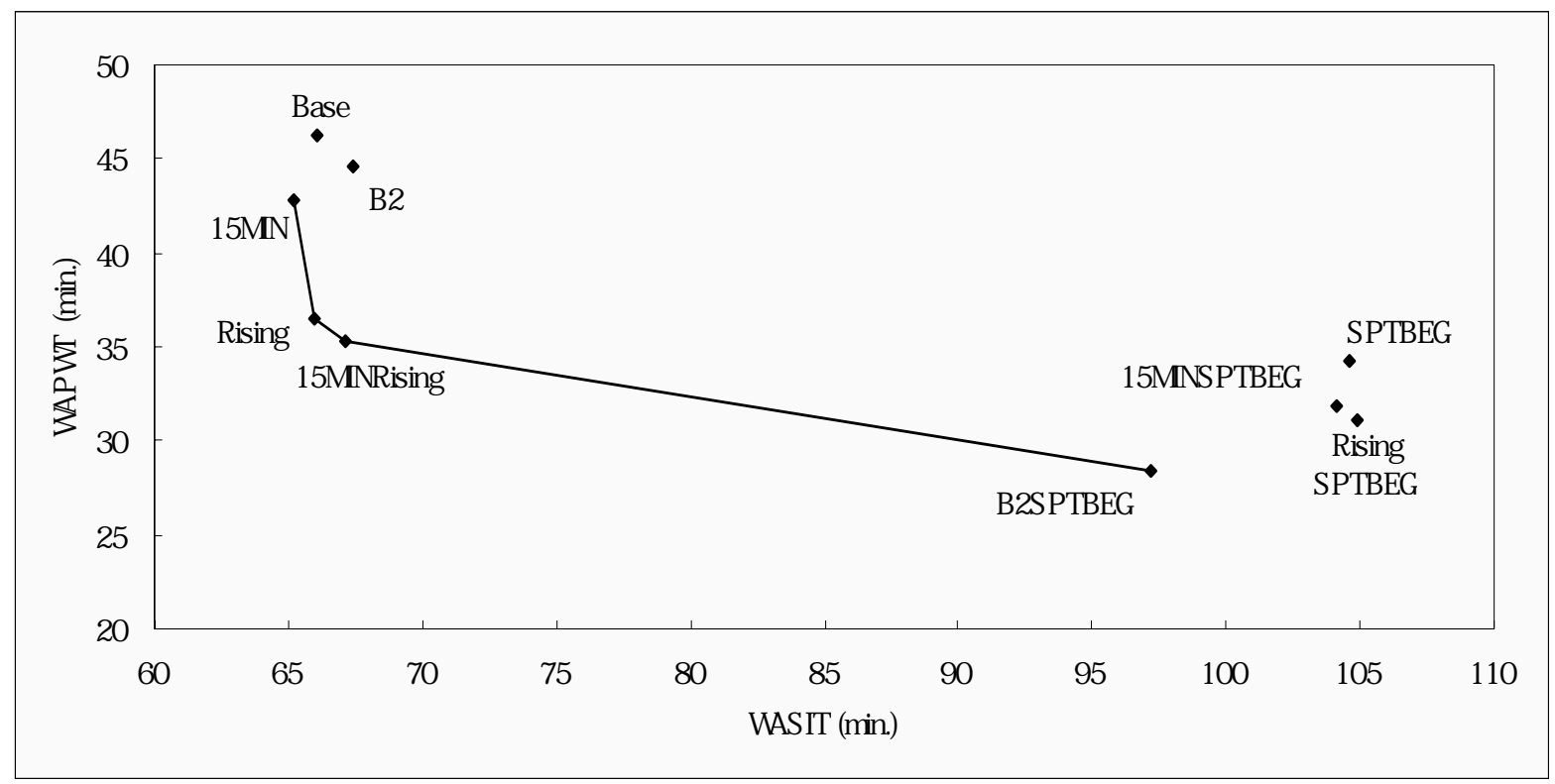

Figure 6: Efficient Frontier.

In all four cases, waiting times of all three types of patients are significantly improved compared to the base case. Compared to Tables IV and V, as expected, adding a physician has a positive effect on both new patients and on non-appointed regular patients. An efficient frontier was drawn using these nine cases with the base case as in Fig. 7. Similar to the preceding frontier, 15MINRising with P2 shows the best option. Yet again, the cases that consist of SPTBEG have bended to the right, due to the high server idle time component of SPTBEG.

Table VI: Waiting time adding a physician for new patients (minutes).

\begin{tabular}{|l|c|c|c|}
\hline \multicolumn{1}{|c|}{ Case } & Appointed & Non-appointed & New \\
\hline Base & 39.13 & 76.55 & 167.05 \\
\hline 15MINP2 & $24.60^{*}$ & $28.65^{*}$ & $90.49^{*}$ \\
\hline SPTBEGP2 & $33.52^{*}$ & $34.10^{*}$ & $91.73^{*}$ \\
\hline RisingP2 & $35.34^{*}$ & $33.97^{*}$ & $90.16^{*}$ \\
\hline B2P2 & $31.11^{*}$ & $63.76^{*}$ & $85.27^{*}$ \\
\hline P2 & $38.94^{*}$ & $50.93^{*}$ & $92.67^{*}$ \\
\hline 15MINRisingP2 & $31.46^{*}$ & $34.87^{*}$ & $92.02^{*}$ \\
\hline B2SPTBEGP2 & $24.73^{*}$ & $26.59^{*}$ & $79.57^{*}$ \\
\hline RisingSPTBEGP2 & $31.31^{*}$ & $29.29^{*}$ & $93.64^{*}$ \\
\hline 15MINSPTBEGP2 & $31.03^{*}$ & $33.68^{*}$ & $92.19^{*}$ \\
\hline
\end{tabular}

The experimental process concentrated on the issue of excessive waiting time at consultation room, aiming to shorten the patients' waiting time. The best option identified in 
this study drastically reduces patient waiting time in any consultation type as shown in Fig. 8a. Because of this, the number of total waiting patients in consultations in any time of the day has reduced significantly (Fig. 8b).

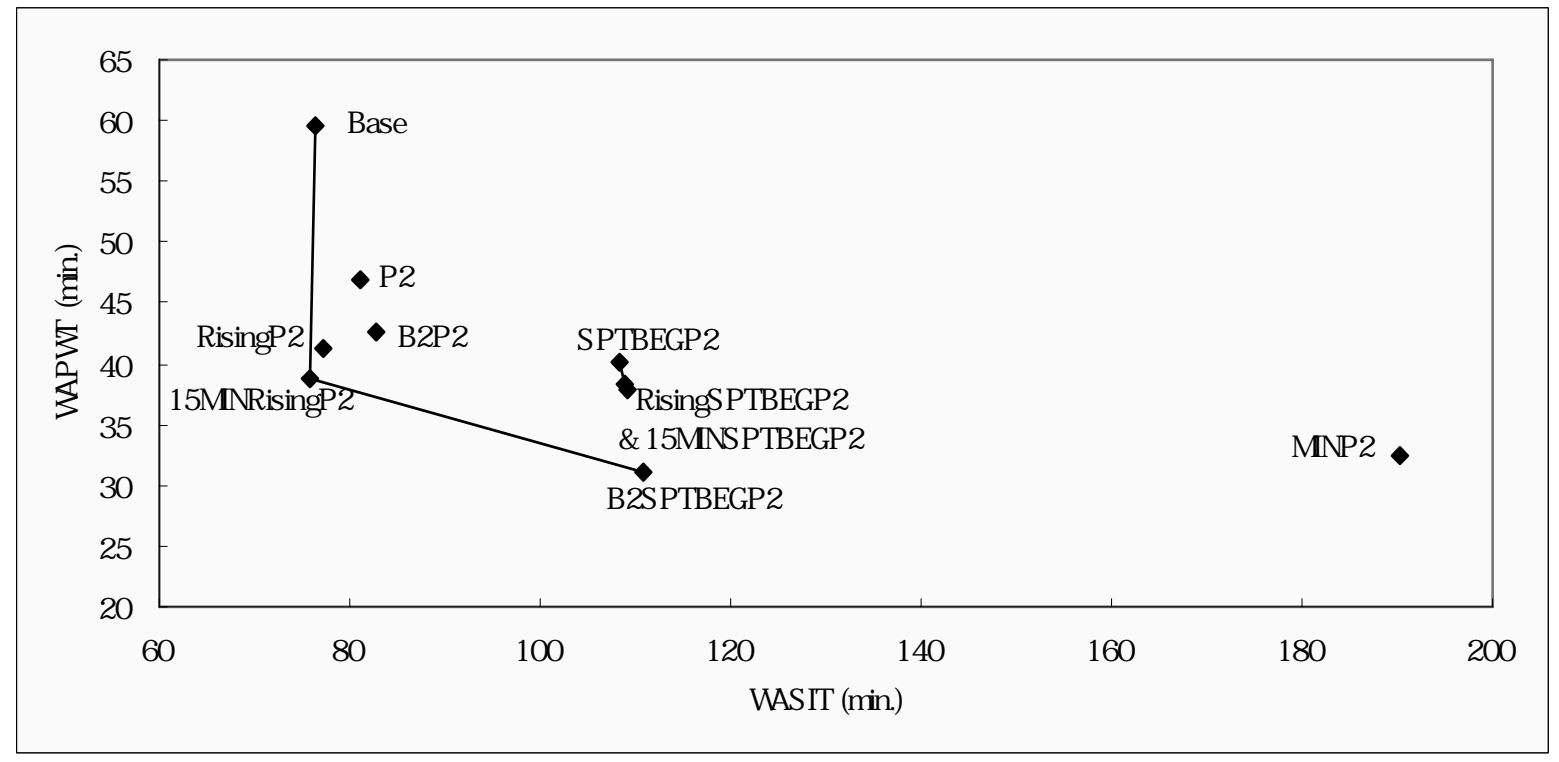

Figure 7: Efficient Frontier including new patients’ category.

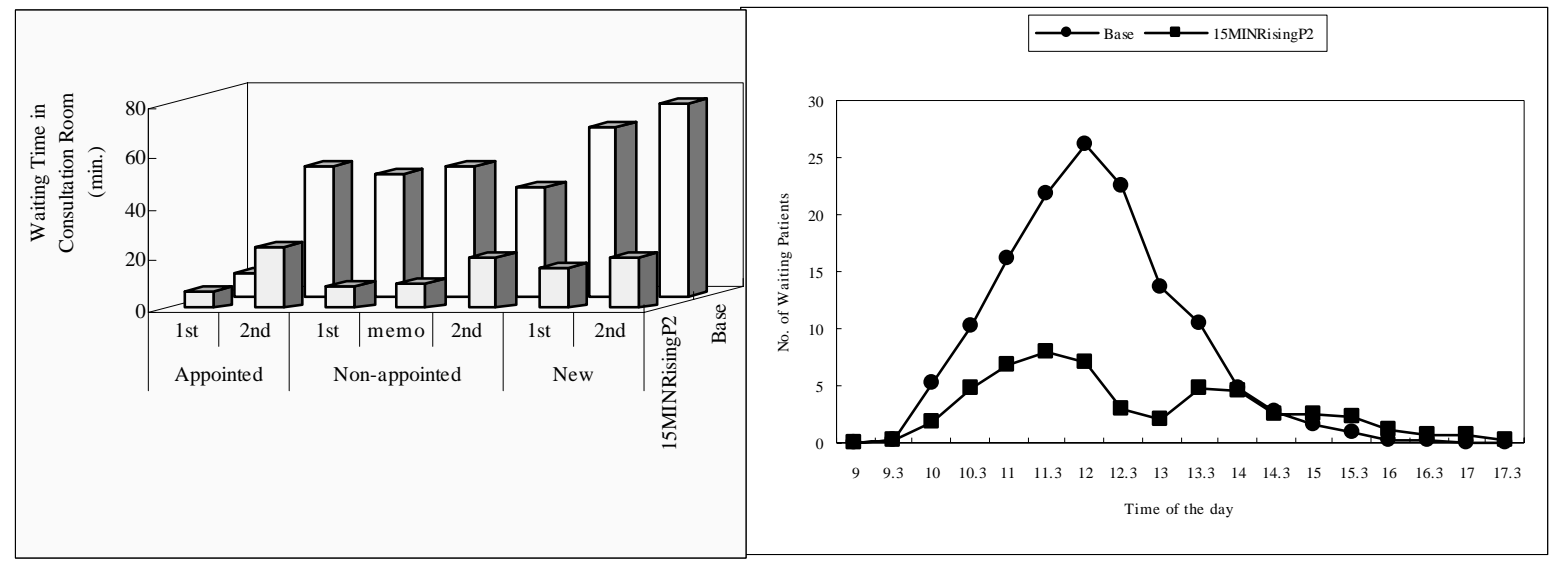

(a) Waiting time of each consultation type

(b) Total waiting patients

Figure 8: Comparison of Base vs. 15MINRisingP2.

A more flattened line depicts the number of waiting patients in consultation rooms for the best case identified in this study. Considering the total number of patients waiting for a day, all three types of patients show a great reduction. The percentage of reduction of the number of waiting patients in consultation rooms for each type was $7 \%, 81 \%$ and $56 \%$ for appointed, non-appointed and new patients respectively. As a result of this, the total number of patients waiting for consultation for a day was reduced from a hundred and thirty six to fifty seven, a 59 percent reduction compared to the existing system. This AS could reduce the total number of patients waiting for consultation at a peak time, from twenty-six to eight, a 69 percent reduction compared to the existing system. 


\section{CONCLUSION}

A simulation analysis was made to examine a number of different ASs on mixed-patients types in the outpatient department of a general hospital. Results show that under the existing system, patients have to wait for a long time for consultations that only last for a few minutes. The experimentation processes concentrated on this issue aiming to shorten waiting time of patients by keeping the idle time of physicians as low as possible.

Four appointment schedule rules and its possible combinations were considered. In the case of appointed and non-appointed patients, it was revealed that the SPTBEG rule is the best in reducing waiting time. The $15 \mathrm{MIN}$ rule is the best in reducing physician idle time. In minimizing both waiting time and idle time the hybrid combination of 15MINRising rule is the best in view of both types of patient category. This rule is still worked best for all types of patient categories, adding one hour capacity to the existing resources for a day by introducing an extra physician to the new patients' care (15MINRisingP2). This option reduces patient waiting time drastically in any consultation type. Moreover, this rule is still worked well in more congested situations at consultation rooms.

Major conclusions in this study:

(1) The popular Baily rule does not perform satisfactorily in actual context;

(2) Both idle time of patients and physicians can be improved by smoothing the demand of scheduling appointment periods to complement the arrival pattern of walk-in patients;

(3) The SPTBEG rule outperforms in terms of patients waiting time; and

(4) A hybrid combination of ASs works well compare to a single AS.

This study ignores non-punctual arrivals and no-show which are common in the outpatients department. Considering these, it would be interesting to investigate the results of scheduling rules employed under the current study. Another area of research is to consider the scheduler uncertainty on SPTBEG rule. Finally it is necessary to do a cost benefit analysis to evaluate the validity of the 15MINRisingP2 rule.

\section{ACKNOWLEDGEMENTS}

The author wishes to express sincere gratitude for Gifu Prefectural Government of Japan for the acknowledgment of this study.

\section{REFERENCES}

[1] Babes, M.; Sarma, G. V. (1991). Out-patient queues at the Ibn-Rochd health center, Journal of the Operational Research Society, Vol. 42, No. 10, 845-855

[2] Klassen, K. J.; Rohleder, T. R. (1996). Scheduling outpatient appointments in a dynamic environment, Journal of Operations Management, Vol. 14, No. 2, 83-101

[3] Klassen, K. J.; Rohleder, T. R. (2004). Outpatient appointment scheduling with urgent clients in a dynamic, multi-period environment, International Journal of Service Industry Management, Vol. 15, No. 2, 167-186

[4] Baily, N. T. J. (1952). A study of queues and appointment systems in hospital out-patient departments, with special reference to waiting-times, Journal of the Royal Statistical Society, Vol. 14, No. 2, 185-199

[5] Soriano, A. (1966). Comparison of two scheduling systems, Operations Research, Vol. 14, No. 3, 388-397

[6] Cox, T. F.; Birchall, J. F.; Wong, H. (1985). Optimizing the queuing system for an ear, nose and throat outpatient clinic, Journal of Applied Statistics, Vol. 12, 113-126 
[7] Rising, E. J.; Baron, R.; Averill, B. (1973). A systems analysis of a university-health-service outpatient clinic, Operations Research, Vol. 21, No. 5, 1030-1047

[8] Liu, L.; Liu, X. (1998). Block appointment systems for outpatient clinics with multiple doctors, Journal of the Operation Research Society, Vol. 49, No. 12, 1254-1259

[9] Ho, C.; Lau, H. (1992). Minimizing total cost in scheduling outpatient appointments, Management Science, Vol. 38, No.12, 1750-1764

[10] Yang, K. K.; Lu, M. L.; Quek, S. A. (1998). A new appointment rule for a single-server, multiple-customer service system, Naval Research Logistics, Vol. 45, 313-326

[11] Vanden Bosch, P. M.; Dietz, D. C. (2000). Minimizing expected waiting in a medical appointment system, IIE Transactions, Vol. 32, 841-848

[12] Wang, P. P. (1993). Static and dynamic scheduling of customer arrivals to a single-server system, Naval Research Logistics, Vol. 40, 345-360

[13] McGuire, F. (1998). Simulation in Healthcare, Banks, J. (Editor), Handbook of Simulation, John Wiley \& Sons, Inc., New York, 605-627

[14] Kelton, W. D.; Sadowski, R. P.; Sturrock, D. T. (2004). Simulation with Arena, McGraw-Hill, New York

[15] Takakuwa, S.; Shiozaki, H. (2004). Functional analysis for operating emergency department of a general hospital, Proceedings of the 2004 Winter Simulation Conference, 2003-2011

[16] Wijewickrama, A. K. A. (2004). Optimum number of parking spaces in a hospital: A simulation analysis, International Journal of Simulation Modelling, Vol. 3, No. 4, 132-141

[17] Law, A. M.; Kelton, W. D. (2000). Simulation Modeling and Analysis, McGraw-Hill, New York 